\title{
Integrating Kano Model, AHP, and QFD Methods for New Product Development Based on Text Mining, Intuitionistic Fuzzy Sets, and Customers Satisfaction
}

\author{
Ming Li $(\mathbb{D})$ and Jie Zhang $(\mathbb{D}$ \\ East China University of Science and Technology, 130 Meilong Road, Xuhui District, Shanghai 200237, China \\ Correspondence should be addressed to Jie Zhang; hdlghd@163.com
}

Received 6 August 2020; Revised 2 February 2021; Accepted 15 March 2021; Published 31 March 2021

Academic Editor: Wen Tsao Pan

Copyright (c) 2021 Ming Li and Jie Zhang. This is an open access article distributed under the Creative Commons Attribution License, which permits unrestricted use, distribution, and reproduction in any medium, provided the original work is properly cited.

\begin{abstract}
Online reviews are crucial to any online business that wants to increase sales on the Internet. Customer reviews have information about product attributes, customer requirements (CRs), and shopping experience; mining reviews provide the direction of decision-making for new product development and design (NPDD). Besides, the information of customer preference has vagueness and uncertainty, and the accuracy of decision-making information directly affects the success of NPDD. This paper proposed a methodology that integrates the Kano model (KM), analytic hierarchy process (AHP), and quality function deployment (QFD) methods with intuitionistic fuzzy set (IFS) to solve decision-making problems in NPDD. By the new method, the web crawler technology was first applied to e-commerce web sites to collect raw data, and the representative CRs were extracted through combining LDA model with Apriori algorithm. Second, the intuitionistic fuzzy Kano model (IFKM) is proposed to evaluate adjustment coefficient of CRs and Kano categories via customer preference membership functions. Thirdly, overall weights which contained emotional needs (ENs) and functional needs (FNs) are obtained via intuitionistic fuzzy analytic hierarchy process (IFAHP); thus, the adjusted weights are calculated from IFKM and IFAHP. Next, the intuitionistic fuzzy quality function deployment (IFQFD) is proposed to acquire engineering characteristics (ECs) of weights through combining competition benchmarks and based on technical benchmarks to make goals for a company's NPDD. Finally, the method was applied to study vertical-configured air conditioner (VAC) as an example. The results showed that the application of text mining and IFS to improve CS is both reliable and scientific.
\end{abstract}

\section{Introduction}

As market competition intensifies and product life cycles constantly get shorten, new product design and development (NPDD) methodology has become an effective way for enterprises to respond to market competition. With the rapid development of science and technology, NPDD also becomes a comprehensive process of diversification and complication. Anderson and Mittal [1] found that, for every $1 \%$ increase in customer satisfaction (CS) with products, the corresponding average return on investment (ROI) is increased by $2.37 \%$, whereas, for every $1 \%$ decrease in satisfaction, the average ROI is decreased by $5.08 \%$. Therefore, improving CS in product development is the key to enterprises' survival and success in the fierce market competition. At the same time, the rapid development of the Internet has provided unprecedented development opportunities for e-commerce platforms; more and more customers purchase products from online and generate huge comments, which contain information like customer needs and product attributes [2]. So, mining customer reviews can provide a scientific direction for NPDD. Moreover, Newman and Patel [3] mentioned three types of product attributes that are associated with customer requirements (CRs), namely, the attributes of basic function, the attributes of convenient function, and the attributes of psychological satisfaction. Both attributes of basic function and convenient function belong to customer functional requirements, and 
the attributes of psychological satisfaction are the higher psychological response of customer experience; that is, the product forms will further promote CS after functions meet customer's basic requirements, thereby resonating with the customer's psychology. In today's environment in which CRs are diversified and personalized, emotional needs (ENs) become increasingly important in customer decision-making to purchase products. This requires NPDD not only to fulfill the customers' basic function needs (FNs) for products, but also to satisfy customers ENs. Therefore, enterprises should pay attention to both ENs and FNs in NPDD.

In recent years, many scholars use the Kansei engineering methodology to explore customer emotional factors and to help companies better understand customer ENs [4]. The Kansei engineering is a product development model originally proposed by Nagamachi. The model is used to quantify Kansei impression based on CRs, thus allowing ones to analyze the relationship between human impression and products forms to determine the right direction of product development [5]. However, Kansei engineering model primarily focuses on directing product form design and lacks an effective approach to assist engineering characteristics (ECs) development. In addition, it is essential to obtain accurate CRs and their categories in the process of CS-oriented NPDD, which determines the success/failure of new product development. The Kano model proposed by Professor Noriaki Kano can solve the problem of CRs' category classification and prioritization. The analysis of the impact of CRs on CS indicates a nonlinear relationship between product performance and CS [6]. But information on human perception and preference is often vague and uncertain. Some scholars have proposed combining fuzzy mathematic and Kano model to transform qualitative analysis into a quantitative one to obtain the preference information of interviewees. However, fuzzy Kano model can only express customer's positive and negative information and cannot deal with customer's uncertainty and hesitation. At last, the quality function deployment (QFD) technique establishes the relationship between the voice of customer (VOC) and the voice of technology (VOT); ENs and FNs are crucial to the improvement of CS [7], because the product form is the first avenue for customer perception to form emotional cognition, then continuously deepening the experience through the use of product functions to fuse the final CS. But enterprises tend to pay more attention to functionality and utility in NPDD, so CRs should be divided into ENs and FNs. Thus, those qualities can be embedded into a product at an early design stage which help companies save time and money, shorten product development cycles, and improve product market competitiveness.

In summary, to improve CS in NPDD process, one will face three key issues: how to get CRs from customer reviews, how to accurately investigate and obtain CRs' preference information, and how to efficiently and accurately improve CRs. In our study, we deal with those issues in several steps. First, we applied Kansei image to express customer's emotional factors (adjective words), combined with topic model and Apriori association algorithm to mine web reviews to obtain CRs including ENs and FNs. Secondly, we conducted questionnaire survey of two-dimensional CS through a combination of the intuitionistic fuzzy set (IFS) and Kano model to obtain customer intuition preference information (positive, negative, and hesitant) and thus to determine CRs categories and adjustment coefficient. Thirdly, we applied intuitionistic fuzzy analytic hierarchy process (IFAHP) to determine the intuition preference relations via the pairwise comparison between each CRs criterion and subcriterion obtained through expert interview. And then, each CRs adjusted importance can be determined by intuitionistic fuzzy Kano model (IFKM) and IFAHP. Lastly, we combined IFS with QFD to gain strong and weak relationships of CRs with ECs and surveyed competition benchmarks to obtain absolute importance of CRs and technical benchmarks to establish EC goals that could be used to guide companies and designers in product development and design optimization.

\section{Literature Review}

2.1. Kansei Engineering. Kansei is a Japanese word, it means "consumer's psychological feeling and image" when translated into English. Kansei engineering is a model for translating feelings and impressions into product parameters. The model was invented in the 1970s by Professor Nagamachi at Kure University. He recognized that companies usually want to quantify the customer's impression of their products. Kansei engineering can measure the feelings and show the correlation to certain product features. Therefore, products can be designed in a way that responds to the intended feeling. Many applications of Kansei engineering focus on the physical design of products appearance. For example, Yang et al. [8] applied rule-based inference model to design telephone's form and style under the framework of Kansei engineering. Yang [9] used a hybrid Kansei engineering system of multiple images for design form of mobile phone. Shieh and Yeh [10] established the relationship between the shape of running shoe and the emotional response of consumers using Kansei engineering methodology, showing that the appearance of running shoe has a significant impact on consumers' overall perception, and their results provided effective guidance for designer in shoe design. Wang [11] combined Kansei engineering with conjoint analysis to achieve product customization of digital cameras and provided decision support for the development of next-generation digital cameras. However, Kansei engineering is rarely used to guide product functions design; most of its applications focus on the psychological impact of product appearance on customers' psychological needs. In addition, it only reflects the corresponding customer's feelings in the process of extracting customer image features and cannot really reflect the customer's demand preferences. Therefore, the current Kansei engineering method has limitations in the analysis of CS with product design.

2.2. Topic Model. The topic model is a statistical model for discovering abstract "topics" that occur in a collection of documents in machine leaning and natural language 
processing. In 1998, Papadimitriou first proposed an early topic model named latent semantic indexing (LSI) [12]. Then, Hofmann created probabilistic latent semantic indexing (PLSI) in 1999 [13]. After continuous research and improvement, latent Dirichlet allocation (LDA) becomes the most common topic model currently in use that is a generalization of PLSI. Developed by David Blei and his colleagues [14] in 2003, LDA model is a Bayesian probability model, which is an unsupervised learning algorithm. Its basic points are words that often show different probability distribution characteristics in different potential topic distributions and the probability of words is relatively close under the same topic. Now, LDA model is usually applied in mining topics, text classification, and text similarity. For instance, Walter et al.[15] used LDA to extract topics from Twitter comments and distinguish man-made accounts. Maier et al.[16] proposed a reliable and effective method with LDA in communication field. Wei et al.[17] created improved LDA to cluster texts. However, there may be a single review involving multiple CRs, and different customers have different idiomaticity that means the same CR was described by different expressions. In addition, a single document often contains multiple topics and topic distributions will change depending on the document. Therefore, based on the LDA model, this paper introduced Apriori association algorithm to solve the above problems.

2.3. Apriori Association Algorithm. Apriori was proposed by Agrawal in 1994 to solve market basket problem. Its purpose is to discover the association rules between different commodities in transaction database [18]. The algorithm uses iterative method of layer-by-layer search to find the relationship between item set to form rules in database, and the concept of item set is the set of items (commodities). If candidate item set meets the minimum support degree, then it is called frequent item set. Now, Apriori algorithm is widely used in business, mobile communication, network security, and management; for example, Guo et al.[19] applied Apriori algorithm to improve the recommendation system of e-commerce platform. Panjaitan et al.[20] used Apriori algorithm to explore consumption pattern. Liu et al.[21] applied Apriori algorithm to solve enterprise stock management problem. Therefore, we attempt to mine the list of topic words as transaction which is got by LDA model and analyze the frequently matched vocabulary with attribute words, thus finding out the content related to these attributes, and finally locate CRs information.

2.4. Kano Model. In 1984, Professor Noriaki Kano and his colleague Fumio Takahashi [22], inspired by Herzberg's motivator-hygiene theory, proposed attractive quality and must-be quality theory. Then, Kano divides quality attributes into five categories: attractive quality, one-dimensional quality, must-be quality, indifferent quality, and reverse quality (Figure 1). The pair of questionnaire surveys, one assumed to be with and another one without quality (CRs) attributes, is conducted to provide insight into which quality attributes fall into which quality categories (Table 1), and it

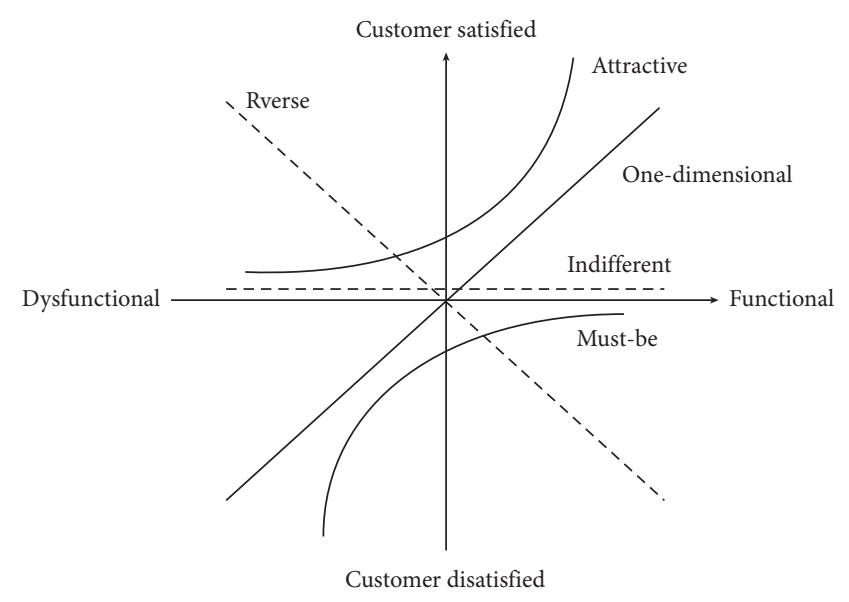

Figure 1: Kano model.

TABle 1: The Kano evaluation table.

\begin{tabular}{lccccc}
\hline Pref. & \multicolumn{5}{c}{ Dysfunctional } \\
Functional & 1. Like & $\begin{array}{c}\text { 2. Must- } \\
\text { be }\end{array}$ & 3. Neutral & $\begin{array}{c}\text { 4. Live } \\
\text { with }\end{array}$ & 5. Dislike \\
\hline 1. Like & Q & A & A & A & $\mathrm{O}$ \\
2. Must-be & $\mathrm{R}$ & $\mathrm{I}$ & $\mathrm{I}$ & $\mathrm{I}$ & $\mathrm{M}$ \\
3. Neutral & $\mathrm{R}$ & $\mathrm{I}$ & $\mathrm{I}$ & $\mathrm{I}$ & $\mathrm{M}$ \\
4. Live with & $\mathrm{R}$ & $\mathrm{I}$ & $\mathrm{I}$ & $\mathrm{I}$ & $\mathrm{M}$ \\
5. Dislike & $\mathrm{R}$ & $\mathrm{R}$ & $\mathrm{R}$ & $\mathrm{R}$ & $\mathrm{Q}$ \\
\hline
\end{tabular}

Note: A: attractive quality; O: one-dimensional quality; M: must-be quality; I: indifferent quality; R: reverse quality; Q: questionable quality.

offers a better understanding of how customers evaluate a product; thus this can be used to assist enterprises to focus on the improvement of most important attributes. After that, the Kano model is widely used to do research by many scholars in NPDD, such as Matzler and Hinterhuber [23], who discussed the strategic importance of the Kano model in NPDD, and proposed the satisfaction coefficient to identify contributions to CS. Tan and Shen [24] incorporated the Kano model into the QFD planning matrix to help understand VOC accurately and deeply and proposed a method of adjusting the importance of CRs by approximating transformation function. Xu et al. [25] attempted to quantify the Kano two-dimensional questionnaire scores in the gradient range of -0.5 to 1 and then converted their values to polar coordinate to get the classification of Kano. The above research on the improvement of the Kano model has moved from qualitative to quantitative level.

Some scholars have tried to study the ambiguity and uncertainty in the process of evaluating human information. For example, Foldesi et al. [26] tried to apply the fuzzy and Kano model to obtain the maximum CRs with financial constraints. Lee and Huang [27] developed the fuzzy Kano two-dimensional questionnaire method, which converts one answer of the traditional Kano questionnaire into multiple answers in percentages. Each percentage represents the membership degree of the standard answer. Although the above-mentioned Kano models with incorporation of fuzzy theory have led to more accurate VOC in expressing uncertainty and ambiguity of CRs, these two methods are still 
of qualitative analysis and do not consider continuous problems. To solve the continuous problems, $\mathrm{Wu}$ and Wang [28] developed a continuous fuzzy Kano model (CFKM) to analyze the ambiguity of CRs more accurately and proposed the influence value matrix to adjust the positive and negative emotional differences in the survey. However, CFKM only describes positive and negative information of customer preference and cannot express hesitation information. Consumers' perception differs according to their experience, knowledge, and environment, which results in different degrees of decision hesitation among attributes. IFs not only describe the degree of membership and nonmembership of a certain preference, but also reflect the degree of hesitation.

Therefore, in this paper, we attempt to obtain the relationship between CRs and CS based on IFs and then help companies analyze consumer preferences more accurately and efficiently in NPDD.

\subsection{Intuitionistic Fuzzy Analytic Hierarchy Process.} Analytic hierarchy process (AHP) is a decision-making tool combining qualitative and quantitative methods, was first proposed by Professor Saaty [29], and has been widely used to solve multicriteria decision-making problems. AHP uses five steps to calculate the combined weight of all constituent elements of each level to the goal: defining goal or problem, establishing hierarchy structure, constructing judgment matrix, sorting single hierarchy, and sorting total hierarchy, thus obtaining the comprehensive evaluation value of different feasible schemes, which provides a basis for choosing the best. However, the key of AHP is to establish a judgment matrix. However, whether the judgment matrix is scientific and reasonable will directly affect the analysis results, and subjectivity and ambiguity of decision-makers in the scoring process always exist. Van Laarhoven and Pedrycz [30] combined fuzzy and AHP to express the ambiguity of decision-makers as triangular fuzzy numbers to solve vagueness problem. Kwiesielewicz [31] mentioned that van Laarhoven and Pedrycz's fuzzy AHP has issues of normal equation having one or more degrees of freedom and applied the generalized pseudoinverse approach to find a structure of general solution. Boender et al. [32] modified van Laarhovern and Pedrycz's fuzzy method and proposed a more robust approach to the normalization of the local priorities. However, these studies have not solved the problem of affirmation, negation, and hesitation of complex cognition, because the membership function used is only single-valued function which cannot be applied to express the support and objection information. Xu and Liao [33] proposed intuitionistic fuzzy analytic hierarchy process (IFAHP) to solve positive, negative, and uncertain preference; besides AHP and FAHP need to reinvestigate the experts when judgment matrix is inconsistent. IFAHP only needs to achieve consistency through the conversion of the judgment matrix. This method also saves time and resources. In addition, the multiattribute decision-making tool represented by AHP has been widely used in NPDD [34-36]. In consequence, this paper used IFAHP to take CS as the target and construct subgoal of customers' ENs and FNs and then obtain the overall weights of CRs, thus multiplying intuitionistic fuzzy Kano evaluation value. In this way, CRs overall adjusted evaluation weights and priority can be obtained.

2.6. Quality Function Deployment. The concept of quality function deployment (QFD) was proposed by Yoji Akao in Japan in the 1960s [37], and this process was integrated with other improvement tools to generate a lot of opportunities for product designers. QFD is a customer-oriented product development tool that achieves higher CS by translating CRs into design requirements, part features, or production plans. The intents of applying QFD are to incorporate VOC into the product development cycle through customer surveys and interviews and to assume the achievement of CS quality. Within the elements of QFD, House of Quality (HOQ) is the core framework of QFD to organize VOC with VOT, and the VOC is expressed in the customer's terms which can be in the form of linguistic or crisp variables. According to research by Hauser and Clausing [38], QFD can reduce product development time by $50 \%$ and cost by $30 \%$. Lai et al. [39] took advantage of Kano model in CRs and combined it with QFD to direct new product development. Tu et al. [40] combined AHP with QFD to develop a sport headphone. Gül Bayraktaroğ and Özge Özgen [41] integrated Kano model, AHP, and QFD to analyze CRs of library services to improve service quality in market strategy development and provide guidance library managers on how to allocate budget, arrange services, and develop their marketing strategies. In the face of vagueness and uncertainty of VOC, Khoo and Ho [42] proposed a fuzzy QFD model, which expresses VOC as a triangular fuzzy number and applied it to the design and development. Wu [43] developed fuzzy HOQ and fuzzy QFD for fuzzy regression evaluation. Similarly, there are characteristics of affirmation, negation, and hesitation in linguistic relation (weak correlation, medium correlation, and strong correlation) in fuzzy HOQ. The membership function cannot fully describe the essential information. So, our study tried to combine IFS and QFD to cover experts' preference information; thus we can achieve engineering characteristics ranking and weights, to finally get goals in NPDD according to the technical benchmarks.

\section{Research Methodology}

3.1. Intuitionistic Fuzzy Set Background. Fuzzy set theory (FS) is an effective technical method for modeling inaccuracy and uncertainty in the real world. FS and IFS are often used to express uncertain information in multiattributes decision-making processes, which has resulted in a great deal of research achievement [44-46]. IFS is an extended fuzzy information concept proposed by Atanassov [47] in 1983 on the basis of FS theory. It extends FS from a single scale to two scales to describe the ambiguity of a physical phenomenon, which possesses three fuzzy states of support, neutrality, and opposition. It has the advantage over FS in accuracy, flexibility, and comprehension. 
Definition 1. Let a fixed universe crisp set $X$ be fixed and let $A \subset X$ be a fixed set [47-49]. An IFS $\widetilde{A}$ in $X$ is an object of the following form:

$$
\widetilde{A}=\left\{\left(x, \mu_{A}(x), v_{A}(x)\right) \mid x \in X\right\},
$$

where the functions $\mu_{A}: X \longrightarrow[0,1]$ and $v_{A}: X \longrightarrow[0,1]$ represent, respectively, the degree of membership and nonmembership of elements $x \in X$ to the set $A$. And, for every $x \in X, 0 \leq \mu_{A}+v_{A} \leq 1$ holds.

Definition 2 [50-52] Triangular intuitionistic fuzzy number (TIFN) $\tilde{a}=\left((a, a, \bar{a}) ; w_{\tilde{a}}, w_{a}\right)$ obviously is a special case of IFS on a real number set $R$, whose membership function and nonmembership function are defined as follows [47-49]:

$$
\begin{aligned}
& \mu_{\tilde{a}}(x)= \begin{cases}(x-\underline{a}) w_{\tilde{a}} /(a-\underline{a}), & \text { if } a \leq x<a, \\
w_{\bar{a}}, & \text { if } x=a, \\
(\bar{a}-x) w_{\tilde{a}} /(\bar{a}-a), & \text { if } a<x \leq \bar{a}, \\
0, & \text { if } x<\underline{a} \text { or } x>\bar{a},\end{cases} \\
& v_{\tilde{a}}(x)= \begin{cases}{\left[a-x+u_{\tilde{a}}(x-\underline{a})\right] /(a-\underline{a}),} & \text { if } a \leq x<a, \\
u_{\tilde{a}}, & \text { if } x=a, \\
{\left[x-a+u_{\tilde{a}}(\bar{a}-x)\right] /(\bar{a}-a),} & \text { if } a<x \leq \bar{a}, \\
1, & \text { if } x<\underline{a} \text { or } x>\bar{a} .\end{cases}
\end{aligned}
$$

The values $w_{a}$ and $u_{a}$ represent the maximum and the minimum degree of nonmembership, respectively, and satisfy the conditions $0 \leq w_{a} \leq 1,0 \leq w_{a} \leq 1$, and $0 \leq w_{\tau^{+}}^{+}$ $u_{a} \leq 1$.

Definition 3. For each IFS $\widetilde{A}$ on $X[47-49]$, then

$$
\pi_{A}(x)=1-\mu_{A}(x)-v_{A}(x),
$$

is called the degree of nondeterminacy (uncertainty) of the membership of the element $x \in X$ to the set $A$. In the case of ordinary fuzzy sets, $\pi_{A}(x)=0$ for every $x \in X$.

Szmidt and Kacprzyk [53] pointed out that $\pi_{A}(x)$ is a hesitancy degree of $x$ to $A$ and cannot be omitted when calculating the distance between two IFSs. For convenience, $\mathrm{Xu}$ [54] called $\alpha=\left(\mu_{\alpha}, v_{\alpha}, \pi_{\alpha}\right)$ an intuitionistic fuzzy value (IFV), where $\mu_{\alpha} \in[0,1], \nu_{\alpha} \in[0,1], \pi_{\alpha} \in[0,1]$, and $0 \leq \mu_{\alpha}+\nu_{\alpha} \leq 1$, and assume that $\Psi$ be the set of all IFVs.

In order to rank the IFVs, Szmidt and Kacprzyk [53] developed a function, defined mathematically as follows:

$$
\rho(\alpha)=0.5\left(1+\pi_{\alpha}\right)\left(1-\mu_{\alpha}\right) .
$$

The smaller the value of $\rho(\alpha)$ is, the greater the IFV $\alpha$ is in the sense of the amount of positive information included and reliability of information.

Definition 4. Let $\widetilde{A}$ and $\widetilde{B}$ be two IFSs in universe $X$ and let $\lambda$ be a real number and $\lambda>0[55]$. The arithmetical operations are stipulated as follows:

$$
\begin{aligned}
\widetilde{A} \oplus \widetilde{B}= & \left\{\left(x, \mu_{A}(x)+\mu_{B}(x)\right.\right. \\
& \left.\left.-\mu_{A}(x) \mu_{B}(x), v_{A}(x) v_{B}(x)\right) \mid x \in X\right\}, \\
\widetilde{A} \otimes \widetilde{B}= & \left\{\left(x, \mu_{A}(x) \mu_{B}(x), v_{A}(x)+v_{B}(x)\right.\right. \\
& \left.\left.-v_{A}(x) \nu_{B}(x)\right) \mid x \in X\right\}, \\
\lambda \widetilde{A}= & \left\{\left(x, 1-\left(1-\mu_{A}(x)\right)^{\lambda},\left(\nu_{A}(x)\right)^{\lambda}\right) \mid x \in X\right\}, \\
\widetilde{A}^{\lambda}= & \left\{\left(x,\left(\mu_{A}(x)\right)^{\lambda}, 1-\left(1-v_{A}(x)\right)^{\lambda}\right) \mid x \in X\right\} .
\end{aligned}
$$

3.2. Selecting Representative Customer Needs. CRs attributes determine the direction of product development process; thus selecting and using improper CRs information could waste companies' large amount of time and money. Thus, to accurately obtain CRs is the first step to successfully develop products. In recent years, many e-commerce platforms such as Amazon and JD.com have launched online review systems for their products or services. Those platforms allow consumers to share and acquire more product information through online reviews. A huge amount of customer comments can be extracted by web crawler technology in e-commerce platform, and ENs and FNs are mined with LDA model and Apriori association algorithm.

The LDA model is represented as probabilistic graphical model in Figure 2 [14]; $\alpha$ is the Dirichlet prior parameter of the topic distribution in each document, reflecting relative strength of the hidden topics in the corpus; $\beta$ is the Dirichlet prior parameter of the word distribution in each topic, reflecting the probability distribution of all hidden topics. And LDA model has two basic assumptions: each document is a set of series of topics and each word belongs to a topic in the document.

The analysis process of LDA model mainly consists of two parts: generative process and parameter estimation. In the generative process part, LDA assumes a corpus $D$ consisting of $M$ documents each of length $N_{i}$ and $K$ denotes topic in $D$.

(1) Choose $\theta_{i} \sim \operatorname{Dirichlet}(\alpha)$, where $i \in\{1,2,3, \ldots, M\}$, $\theta_{i}$ is the topic distribution for document $i$, and Dirichlet $(\alpha)$ is a Dirichlet distribution with a symmetric parameter $\alpha$ which is typically sparse $(\alpha<1)$.

(2) Choose $\varphi_{k} \sim \operatorname{Dirichlet}(\beta)$, where $k \in\{1,2,3, \ldots, K\}$, $\varphi_{k}$ is the word distribution for topic $k$, and $\beta$ is typically sparse.

(3) For each of the word positions $i$ and $j$, where $i \in\{1,2,3$, $\ldots, M\}$ and $j \in\left\{1,2,3, \ldots, N_{i}\right\}$, we het the following. Then, choose a topic $z_{i j} \sim \operatorname{Multinomial}\left(\theta_{i, k}\right)$, where $z_{i j}$ is the topic for $j$ th word in document $i$ and choose a word $w_{i j} \sim \operatorname{Multinomial}\left(\varphi_{k, t}\right)$, where $w_{i j}$ is the specific word; multinomial distribution refers to the multinomial with only one trial, which is also known as the categorical distribution.

In the parameter estimation part, through the estimation of $\varphi$ and $\theta$, we can obtain the relevant information of the 


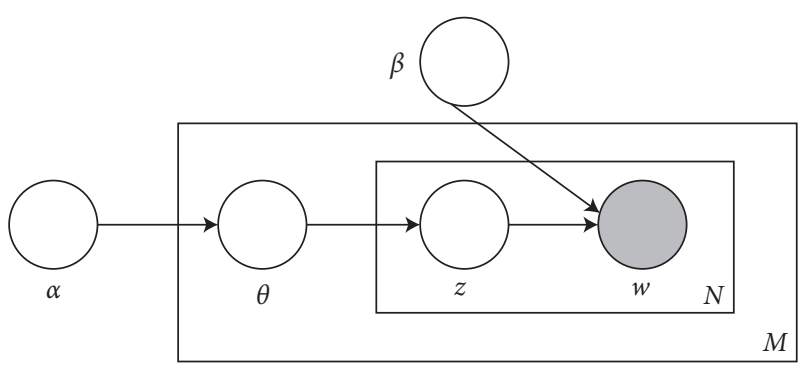

FIgURE 2: Graphical model representation of LDA.

topics contained in the corpus and its proportion in each document. These parameters using Gibbs sampling [56] are estimated as follows:
(1) Estimate the probability value of a document.

$$
p(w \mid \alpha, \beta)=\int p(\theta \mid \alpha)\left(\prod_{j=1}^{N} \sum_{z_{j}} p\left(z_{j} \mid \theta\right) p\left(w_{j} \mid z_{j}, \beta\right)\right) d \theta .
$$

Collapsed Gibbs sampling first estimates the relationship between each word and topic and then calculates these parameters through frequency statistics.

(2) Calculate the conditional probability of the topic sequence under the word sequence.

$$
p\left(z_{j}=k \mid z_{-j}, w\right)=\frac{p(w, z)}{p\left(w, z_{-j}\right)} \propto \frac{n_{k,-j}^{t}+\beta_{t}}{\sum_{t=1}^{V} n_{k,-j}^{t}+\beta_{t}} \frac{n_{i,-j}^{k}+\alpha_{k}}{\sum_{i=1}^{K} n_{i,-j}^{k}+\alpha_{k}}
$$

where $z_{j}$ represents the assignments of $j$ th word in a document to topic $k, z_{-j}$ represents all topic assignments not including $j$ th word, $n_{k}^{t}$ denotes the number of times in which word $t$ is assigned to topic $z_{k}$, and $n_{i}^{t}$ denotes the number of times in which topic $z_{k}$ is assigned to document $m_{i}$.

(3) Calculate $\varphi$ and $\theta$ parameters.

$$
\begin{gathered}
\varphi_{k, t}=\frac{n_{k}^{t}+\beta_{t}}{\sum_{t=1}^{V} n_{k}^{t}+\beta_{t}}, \\
\theta_{i, k}=\frac{n_{i}^{k}+\alpha_{t}}{\sum_{t=1}^{K} n_{i}^{k}+\alpha_{t}},
\end{gathered}
$$

where $\varphi_{k, t}$ represents the probability of word $t$ in topic $z_{k}$ and $\theta_{i, k}$ represents the probability of topic $z_{k}$ in document $i$. So, we can get the relations topic-word and document-topic by probability value.

In LDA model process, the perplexity [14] is used to determine the number of topics, and the smaller the perplexity, the better the model performance; see the following formula:

$$
\operatorname{perplexity}(w)=\exp \left\{-\frac{\log (p(w))}{\sum_{i=1}^{N} \sum_{t=1}^{V} n_{i}^{t}}\right\}
$$

where $V$ is the number of words in corpus $D$ and $n_{i}^{t}$ denotes the number of times in which word $t$ is in $i$ th document.

However, there are two issues in mining CRs. Firstly, a single customer review (document) may include multiple CRs; secondly, due to different idiomaticity, the same need is expressed in different ways. Hence, mining words frequently collocate with attribute words; we can find out the content related to these attributes so that CRs are located. In the example, we used the topic words of multiple iterations of the LDA model as transactions.
The Apriori algorithm uses breadth-first search and Hash tree structure to count candidate item sets efficiently [18]. It generates candidate item set of length $k$ from sets of length $k$ 1 , then it prunes the candidates which have an infrequent subpattern, and these sets of items have at least the given minimum support. After that, it scans the transaction database to determine frequent items sets among the candidates. The minimum support and minimum confidence are measured association rules in transaction, which represents certainty and usefulness, respectively, as in (13)-(14). And lift is a supplement to association rules, which reflects the worth of the rules as in (15):

$$
\begin{aligned}
\operatorname{support}(A \Rightarrow B) & =P(A \cup B), \\
\operatorname{confidence}(A \Rightarrow B) & =P(B \mid A)=\frac{\operatorname{support}(A \cup B)}{\operatorname{support}(A)} \\
& =\frac{\operatorname{support} \_\operatorname{count}(A \cup B)}{\operatorname{support} \_\operatorname{count}(A)}, \\
\operatorname{lift}(A \Rightarrow B) & =\frac{\text { confidence }(A \Rightarrow B)}{\operatorname{support}(B)},
\end{aligned}
$$

where $A$ and $B$ represent the item sets (topic words), $A \Rightarrow B$ denote an association rule, greater than minimal support value $S$, and $S$ represents the ratio of all topics that include both topic words $A$ and $B$, which denote $P(A \cup B)$. If $S$ is smaller, it means that the relationship between $A$ and $B$ is not great; otherwise, $A$ and $B$ are related. The confidence degree $C$ represents the probability that the topic of item sets $A$ and $B$ appears at the same time, which denote $P(B \mid A)$. If $C$ is smaller, it means that both $A$ and $B$ have little chance to appear. The lift degree $L$ reflects the correlation between $A$ and $B$ in the association rules. If $L$ is greater than 1 , thus this means higher positive correlation; if $L$ is less than 1 , thus this means higher negative correlation; otherwise, $A$ and $B$ are not relevant. 


\subsection{Intuitionistic Fuzzy Kano Model Acquires CRs Category} and Adjustment Coefficient. The Kano model has been widely used in industry and scientific research. More specifically, it is used to analyze and classify CRs. In addition, the Kano model is a two-dimensional quality model and is used to present asymmetry and nonlinear relationship between product performance and CS [27]. The questionnaire survey (Table 2) includes a pair of questions (function/ dysfunction CRs attributes), which is used to calculate Kano categorization [22]. As mentioned before, information for evaluation, which is solely based on crisp value (classical Kano model) or positive/negative value (FKM and CFKM), is not complete, because people often express their preference in the scoring process with uncertainty and vagueness.

The triangular IFNs (TIFNs) are a special case of fuzzy sets and are important for fuzzy multiattributes decisionmaking problems [51]. Intuitionistic fuzzy Kano model (IFKM) is different from FKM, and it only uses one point in the survey to express customers' preference, and it is also necessary when investigating the scores of satisfaction and dissatisfaction and the need for obtaining positive, negative, and hesitant preference in the questions (Figure 2). Besides, the degree of membership of one's preference to a standard answer (enjoy, expect, neutral, live with, or dislike) can be considered as a decreasing function of the distance between the chosen point and the standard answer. The closer the chosen point to the standard answer, the greater the membership degree of one's preference to it. In this paper, we use triangular membership functions to represent the memberships of one's preference to the five standard answers as follows:

(1) Dislike $(1,1,3)$

$$
f_{R}(x)= \begin{cases}w_{\tilde{a}}, & x=1, \\ \frac{1}{2}(3-x) w_{\tilde{a}}, & 1<x \leq 3 \\ 0, & \text { otherwise. }\end{cases}
$$

(2)Live with $(1,3,5)$

$$
f_{R}(x)= \begin{cases}\frac{1}{2} w_{\tilde{a}}(x-1), & 1 \leq x<3 \\ w_{\tilde{a}}, & x=3 \\ \frac{1}{2}(5-x) w_{\tilde{a}}, & 3<x \leq 5 \\ 0, & \text { otherwise }\end{cases}
$$

(3) Neutral $(3,5,7)$

$$
f_{R}(x)= \begin{cases}\frac{1}{2} w_{a}(x-3) & 3 \leq x<5 \\ w_{\tilde{a}} & x=5 \\ \frac{1}{2}(7-x) w_{\tilde{a}} & 5<x \leq 7 \\ 0 \text { otherwise. }\end{cases}
$$

(4)Expect $(5,7,9)$

$$
f_{R}(x)= \begin{cases}\frac{1}{2} w_{\tilde{a}}(x-5), & 5 \leq x<7, \\ w_{\tilde{a}}, & x=7, \\ \frac{1}{2}(9-x) w_{\tilde{a}}, & 7<x \leq 9, \\ 0, & \text { otherwise. }\end{cases}
$$

(5)Enjoy (7, 9, 9)

$$
f_{R}(x)= \begin{cases}\frac{1}{2} w_{a}(x-7), & 7 \leq x<9, \\ w_{\tilde{a}}, & x=9 .\end{cases}
$$

where $w_{\tilde{a}}$ is the maximum membership of one's attribute and the membership degrees of one's preference to the standard answers can be obtained through the membership functions. For example, the maximum membership and minimum nonmembership are 0.9 and 0 , respectively. And an interviewee chooses the point 3.3 as his/her answer to a certain question; the membership degrees of his/her preference to 'like with' and 'neutral' are 0.765 and 0.135 , respectively (Figure 3).

IFKM uses Kano evaluation table to combine the membership of the function with that of the dysfunction of a certain attribute (Table 1). For instance, one respondent's preference may be assigned to cells $(2,4),(2,5),(1,4)$, and (1, $5)$ in Table 1 . The membership degree $\mu_{n i j}$ of his/her preference combination $(i, j)$ is determined by the following form [27]:

$$
\mu_{n i j}=m\left(F_{i}\right)_{n} \times m\left(D_{j}\right)_{n}
$$

where the subscript $n$ represents $n$th participant; $m\left(F_{i}\right)_{n}$ and $m\left(D_{j}\right)_{n}(i, j=1,2, \ldots, 5)$ are the membership degrees of his/her preference to $i$ th standard answer to the functional questions and $j$ th standard answer to the dysfunctional questions, respectively. Then, the membership degree $\mu_{n i j}$ of $n$th participant's preference can be identified as the degree of "attractive quality," "must-be quality," "one-dimensional quality," "indifferent quality," or "reverse quality."

According to the Kano evaluation in Table 1, 25 combinations contribute different values to CS, because "like" has more intense emotion, and "must-be" has only weaker expression of satisfaction. Similarly, "dislike" is a very strong dislike, whereas "live with" is only a weak expression of dissatisfaction [57]. In addition, Tan and Shen [24] and Chen and Ko [58] suggested assigning values of 2, 1, and 0.5 to the corresponding categories of must-be, one-dimensional, and attractive. Therefore, in the upper-right quadrant of Table 1, the influence values of pure "must-be," "one-dimensional," and "attractive" are 2, 1, and 0.5 , respectively. In lower-left quadrant of Table 1, the answers of the function are stronger responses than dysfunction ones, so the negative influence values are half as large as the corresponding positive ones. Thus, a standard matrix of the influence value was given as follows: 
TABLE 2: The IFKM questionnaire.

Function and dysfunction questions (e.g., if the VAC has/does not have simple and liberal attribute, how do you feel?)

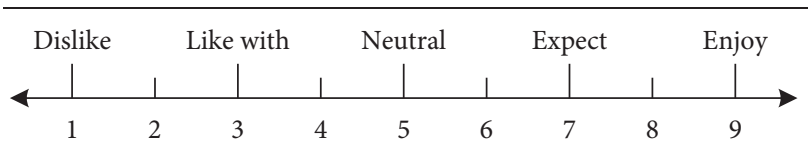

Degree of score satisfaction and dissatisfaction (e.g., what do you feel according to your choice?)

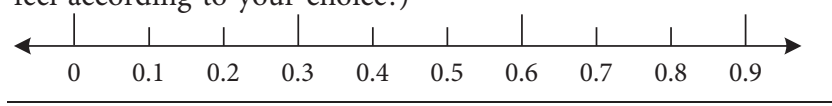

Note. Satisfaction + dissatisfaction is less than 1 .

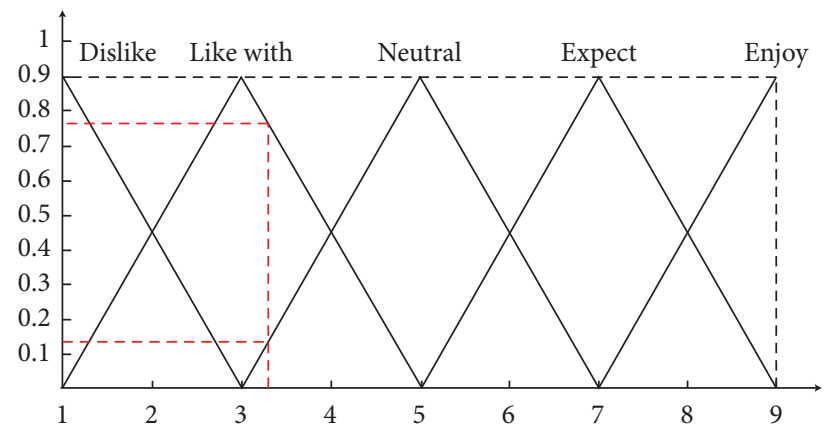

FIGURE 3: Membership functions of IFNs.

$$
v_{i j}=\left[\begin{array}{ccccc}
0 & 0.200 & 0.250 & 0.300 & 0.500 \\
-0.100 & 0 & 0.050 & 0.075 & 0.900 \\
-0.125 & -0.025 & 0 & 0.100 & 1.000 \\
-0.150 & -0.038 & -0.050 & 0 & 0.8 \\
-0.250 & -0.450 & -0.500 & -0.400 & 0
\end{array}\right] \text {. }
$$

At last, $n$th participant's satisfaction value of the quality $S F_{n}$ can be obtained by a mathematical aggregation of all influence values weighted by the corresponding membership degrees.

$$
S F_{n}=\sum_{i=1}^{5} \sum_{j=1}^{5} v_{i j} \times \mu_{n i j} .
$$

\subsection{Intuitionistic Fuzzy Analytic Hierarchy Process to Obtain} ENs and FNs Importance. IFKM can be used to obtain priorities and Kano categories of customers' ENs and FNs, respectively, but the weights of ENs and FNs cannot be compared directly. Because Kansei image and function are different abstract concepts, experts are more comprehensive and professional than customers to distinguish the weight. IFAHP can be considered as an analysis method of extending AHP and FAHP [33], where FAHP can analyze and process the participants' membership information better than AHP, and IFAHP can obtain nonmembership and neutral information better than FAHP. IFAHP analysis includes the following 7 steps:

(1) Identify the objective, criteria, and subcriteria of the decision-making problem and then construct the hierarchy of the considered problem. This step is similar to the classical AHP.

(2) Determine the intuitionistic preference relation via the pairwise comparison between each criterion and subcriterion. And then, the intuitionistic preference relations are constructed. The scale regarding the relative importance degrees is denoted by IFVs.

(3) If all the intuitionistic preference matrices have acceptable consistency, go to the next step. The consistency of each intuitionistic preference matrix is given as follows:

$$
\begin{gathered}
\bar{\mu}_{i k}=\frac{\sqrt[k-i-1]{\prod_{t=i+1}^{k-1} \mu_{i t} \mu_{t k}}}{\sqrt[k-1]{\prod_{t=i+1}^{k-1} \mu_{i t} \mu_{t k}}+\sqrt[k-i-1]{\prod_{t=i+1}^{k-1}\left(1-\mu_{i t}\right)\left(1-\mu_{t k}\right)}}, \quad k>i+1, \\
\bar{v}_{i k}=\frac{\sqrt[k-i-1]{\prod_{t=i+1}^{k-1} v_{i t} v_{t k}}}{\sqrt[k-i-1]{\prod_{t=i+1}^{k-1} v_{i t} v_{t k}}+\sqrt[k-i-1]{\prod_{t=i+1}^{k-1}\left(1-v_{i t}\right)\left(1-v_{t k}\right)}} \quad k>i+1, \\
d(R, \bar{R})=\frac{1}{2(n-1)(n-2)} \sum_{i=1}^{n} \sum_{k=1}^{n}\left(\left|\bar{\mu}_{i k}-\mu_{i k}\right|+\left|\bar{v}_{i k}-v_{i k}\right|+\left|\bar{\pi}_{i k}-\pi_{i k}\right|\right), \\
d(R, \bar{R})<\tau
\end{gathered}
$$

where $\bar{\mu}_{i k}$ is the consistency preference membership; $\bar{\nu}_{i k}$ is consistency preference nonmembership, and $i<k<t(i, k, t=1,2,3, \ldots, n)$ are elements of matrix; $\mu_{i k}$ is original preference membership; $\nu_{i k}$ is original preference membership; $R$ is the expert judgment preference matrix; $\bar{R}$ is consistency judgment matrix; $\tau$ is consistency threshold value and is generally set to 0.1 . 
(4) Recalculate the inconsistent intuitionistic preference relations as follows:

$$
\begin{aligned}
\tilde{\mu}_{i k}^{(p)} & =\frac{\left(\mu_{i k}^{(p)}\right)^{1-\sigma}\left(\bar{\mu}_{i k}^{(p)}\right)^{\sigma}}{\left(\mu_{i k}^{(p)}\right)^{1-\sigma}\left(\bar{\mu}_{i k}^{(p)}\right)^{\sigma}+\left(1-\mu_{i k}^{(p)}\right)^{1-\sigma}\left(1-\bar{\mu}_{i k}^{(p)}\right)^{\sigma}}, \quad i, k=1,2, \ldots, n, \\
\tilde{v}_{i k}^{(p)} & =\frac{\left(v_{i k}^{(p)}\right)^{1-\sigma}\left(\bar{v}_{i k}^{(p)}\right)^{\sigma}}{\left(v_{i k}^{(p)}\right)^{1-\sigma}\left(\bar{v}_{i k}^{(p)}\right)^{\sigma}+\left(1-\nu_{i k}^{(p)}\right)^{1-\sigma}\left(1-\bar{\nu}_{i k}^{(p)}\right)^{\sigma}}, \quad i, k=1,2, \ldots, n, \\
d\left(\bar{R}, R^{(p)}\right) & =\frac{1}{2(n-1)(n-2)} \sum_{i=1}^{n} \sum_{k=1}^{n}\left(\left|\bar{\mu}_{i k}-\mu_{i k}^{(p)}\right|+\left|\bar{v}_{i k}-v_{i k}^{(p)}\right|+\left|\bar{\pi}_{i k}-\pi_{i k}^{(p)}\right|\right),
\end{aligned}
$$

where $p$ denotes the number of iterations and $p \geq 2$; $\widetilde{\mu}_{i k}^{(p)}$ is iterative membership; $\widetilde{\nu}_{i k}^{(p)}$ is iterative nonmembership; $\sigma$ denotes controlling parameter determined by the decision-maker, and $0 \leq \sigma \leq 1$. The smaller the value of $\sigma$, the closer the $\widetilde{R}^{(\bar{p})}$ to $R^{(p)}$.

(5) Calculate the weight vector $K=\left(K_{1}, K_{2}, \ldots K_{n}\right)$ of each intuitionistic preference relation as follows:

$$
\begin{aligned}
w_{i} & =\frac{\sum_{k=1}^{n}\left[\mu_{i k}, 1-v_{i k}\right]}{\sum_{i=1}^{n} \sum_{k=1}^{n}\left[\mu_{i k}, 1-v_{i k}\right]} \\
& =\frac{\left[\sum_{k=1}^{n} \mu_{i k}, \sum_{k=1}^{n}\left(1-v_{i k}\right)\right]}{\left[\sum_{i=1}^{n} \sum_{k=1}^{n} \mu_{i k}, \sum_{i=1}^{n} \sum_{k=1}^{n}\left(1-v_{i k}\right)\right]}, \\
& =\left[\frac{\sum_{k=1}^{n} \mu_{i k}}{\sum_{i=1}^{n} \sum_{k=1}^{n}\left(1-v_{i k}\right)}, \frac{\sum_{k=1}^{n}\left(1-v_{i k}\right)}{\sum_{i=1}^{n} \sum_{k=1}^{n} \mu_{i k}}\right], \quad i=1,2, \ldots, n .
\end{aligned}
$$

Then, we can transform each interval into a corresponding IFV.

$$
W_{i}=\left(\frac{\sum_{k=1}^{n} \mu_{i k}}{\sum_{i=1}^{n} \sum_{k=1}^{n}\left(1-v_{i k}\right)}, 1-\frac{\sum_{k=1}^{n}\left(1-v_{i k}\right)}{\sum_{i=1}^{n} \sum_{k=1}^{n} \mu_{i k}}\right) .
$$

(6) Combine all the ratio of importance from the lowest level to the highest level by the operations of IFVs, to obtain the overall weights $W K_{i}$ using formula (6).

3.5. Intuitionistic Fuzzy Quality Function Deployment to Obtain Engineering Characters Priorities. The impact of ENs on CS is becoming more and more important, but enterprise usually ignores the ENs in the NPDD. And the CRs are divided into ENs and FNs in a way that could effectively improve CS. Furthermore, in the NPDD processing, ENs directly affect product form design, and FNs impact greatly on functional parameter design, because product function matches customer usage needs and product form satisfies customer aesthetic needs. Thus, application QFD finds out the relations of CRs and ECs and establishes the goals of NPDD.

The construction of HOQ is the most important process in implementing QFD (Figure 4), and it consists of CRs input left wall (WHAT), ECs input ceiling (HOW), competition benchmarks input right wall (WHO), CRs and ECs relation matrix input ROOM, ECs correlation matrix input roof (WHY), ECs weights output floor (HOW MANY), technical benchmarks input basement (WHERE), and strategy of NPDD output goals (METHOD). CRs are obtained by LDA model and Apriori algorithm, and the importance of CRs is gained by IFKM and IFAHP (32). HOWs is acquired via expert interview; ROOM is gained through the relationship between VOC and VOT. By the relation symbols proposed by Cohen [59], $\Delta$ denotes weak correlation, Odenotes moderate correlation, and $\odot$ denotes strong correlation. Every EC has to be paired with at least one relation symbol of CRs; otherwise, it should be abandoned, and every corresponding symbol is converted into IFV (Table 3); WHO consists of market competition evaluation, target satisfaction, rate of satisfaction improvement, and absolute weights of CRs, where market competition evaluation relies on five-Likert scale (extreme importance/extreme unimportance) to evaluate CRs; target satisfaction depends on importance of CRs and developers to determine subjectively; rate of satisfaction improvement is obtain using (34); absolute weights of CRs use formula (35); WHY is gained by expert interview, + denotes positive correlation, and - denotes negative correlation; according to (36)-(38); HOW MANY can be obtained; WHERE is acquired by technical developer number through web crawler; METHOD is obtained by developers based on technical competition benchmarks.

$$
\begin{aligned}
W_{i} & =W K_{i} S F_{i}, \\
R s i_{i} & =T s_{i} / C s_{i}, \\
A W_{i} & =W_{i} R s i_{i},
\end{aligned}
$$

where $R s i_{i}$ denotes rate of satisfaction improvement, $T s_{i}$ denotes competitor target satisfaction of CRs, $C s_{i}$ is development company satisfaction of CRs, $W_{i}$ denotes adjusted importance, $W K_{i}$ denotes ratio of importance, $S F_{i}$ denotes adjustment coefficient, $A W_{i}$ denotes absolute weight, and $i=1,2, \ldots$ nis the number of CRs. 


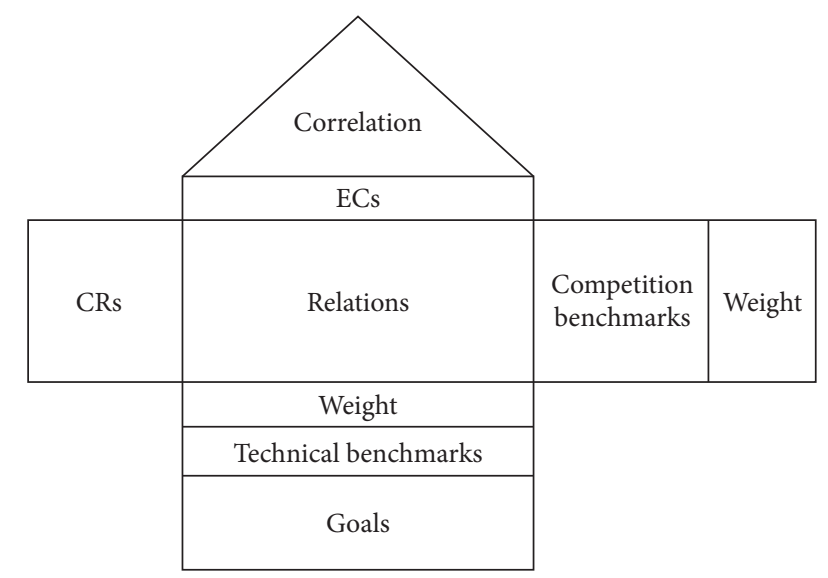

FIGURE 4: Model of QFD procedures.

TABLE 3: Symbols and IFVs in QFD.

\begin{tabular}{lcc}
\hline Symbols & Definition & IFVs \\
\hline Blank & No relationship & $(0.1,0.9)$ \\
$\triangle$ & Weak relationship & $(0.3,0.6)$ \\
$\bigcirc$ & Medium relationship & $(0.6,0.3)$ \\
$\odot$ & Strong relationship & $(0.9,0.1)$ \\
\hline
\end{tabular}

Then, based on HOQ model, we can obtain the standard weight value $E C N_{j}$ of ECs as follows:

$$
\begin{gathered}
E C_{j}=\sum_{i=1}^{n} A W_{i} \otimes S_{i j}, \\
\rho\left(E C_{j}\right)=0.5\left(1+\pi_{E C j}\right)\left(1-\mu_{E C j}\right), \\
E C N_{j}=\frac{\rho(E C)}{\sum_{j=1}^{m} \rho(E C)_{j}},
\end{gathered}
$$

where $S_{i j}$ is CRs and ECs intuitionistic fuzzy relation matrix, $E C_{j}$ is intuitionistic importance of $j$ th WHAT, $\rho\left(E C_{j}\right)$ is the real number of ECs, $E C N_{j}$ is the standard weight, and $i=$ $1,2, \ldots, n$ denotes CRs number, and $j=1,2, \ldots m$ denotes ECs number.

\section{Case Study}

For this section, we use vertical-configured air conditioner (VAC) as a research example. VAC has become essential electrical device for homes and workplaces where they create a comfortable environment for living and work, increasing people's feeling of happiness in their daily life. When purchasing a VAC, customers not only consider the product's basic functions, but also concern about its external form and appearance. Thus, Kansei factors play a critical role in customer's purchasing decision. Since VAC becomes widely available throughout homes and workplaces, people have accumulated lots of experience with the VAC and developed good understanding of the various aspects of the products. Therefore, customers could provide valuable information on the VAC products, which could facilitate our research study. Thus, we could acquire the information on which CRs are more important for influencing customers' decisions of buying the VAC products and how and where companies innovate and prioritize their product design and provide assistance to designers and engineers in product development. In the next section, we will apply the proposed method to the VAC. It should be noted that the method is also applicable to other products.

4.1. Step 1. Mining CRs. We collected customer reviews by Octopus and saved in xls format from e-commerce platform (JD.com), with a result of total 5391 comments in Chinese. The original data was preprocessed, including removing invalid and duplicate comments and deleting punctuation. The Python language is applied throughout the retrieval process. Then, we called the Jieba library for Chinese segmentation and obtained 5310 valid comments. According to perplexity (14) to determine the topic number $K$, we do not estimate the hyperparameters $\alpha$ and $\beta$-instead the smoothing parameters are fixed at $50 / K$ and $200 / M$, respectively, where $K$ is the topic number of LDA models, $M$ is training samples containing $4248(80 \%)$ documents in LDA, and the LDA model tested samples are $1062(20 \%)$. The result of perplexity is shown in Figure 5; the topic number is 90 and the model has better generalization ability.

In consequence, the topic number is 90 ; we used Gensim library to construct LDA model which iterated 6000 through corpus and every 100 iteration to record the topic words. The results (Table 4) do not quite focus on product attributes by LDA model. So, we applied Apriori association algorithm to analyze 60 transactions; each transaction contains 90 topics, and each topic includes 10 topic words.

We obtained 7 strong association rules about sound in Table 5 , and the rules are described in 7 ways meaning the same CR; we can adjust the order of words in these strong association rules to gain easy-to-understand sentence that is low noise.

Similarly, we obtained all the CRs in Table 6 when minimum support set is 0.005 and minimum confidence set is 0.5 , and the CRs of VAC contain 3 ENs and 6 FNs from topic words. But there are massive invalid rules. There may be three reasons. Firstly, since the frequency of words about dehumidification and air purification is quite low in corpus, the LDA model cannot cover these words in priority of 10 topic words. Secondly, the Apriori algorithm prunes frequency item sets (transactions) which is noun and does not consider the relationship with different parts of speech. Thirdly, Chinese segmentation may be inaccurate, because some Chinese words have multiple parts of speech and different order has different meaning.

\subsection{Step 2. Obtaining CRs Category and Adjustment} Coefficient. According to Table 2, we made Kano questionnaire including 9 CRs attributes. 200 interviewees including housewives, white collar workers, and college students (100 males and 100 females), from 20 to 55 years old, were invited. Every participant was required to answer two-dimensional function and dysfunction quality questions and fill with their scores of satisfaction and dissatisfaction. 195 questionnaires were returned, a response rate of 97.5 percent, and 182 questionnaires were reasonable, a 


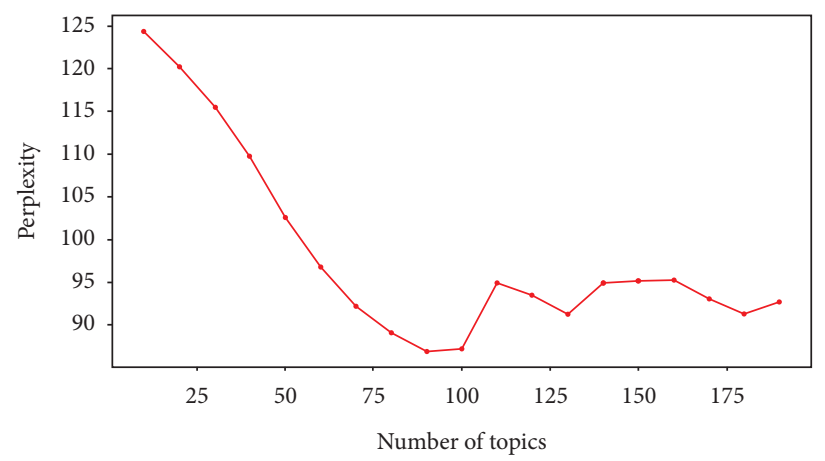

Figure 5: Perplexity on different topics.

TABLE 4: Inductive topics.

\begin{tabular}{lcc}
\hline No. & Summary & Main topics \\
\hline 1 & Function & Sound, energy, cooling, heating, mobile, and wind \\
2 & Form & Beautiful appearance \\
3 & Service & Installation, delivery, and after sale \\
4 & Experience & Shopping and working \\
5 & Price & Double 11,618 \\
6 & Brand & Midea, Gree, and Aux \\
\hline
\end{tabular}

TABLE 5: Example of strong rules.

\begin{tabular}{|c|c|c|c|c|}
\hline rules & Chinese/ & Support & Confide & Lift \\
\hline 声音] [c & 听不 & & & 12.41 \\
\hline$=>$ [声音] $[\mathrm{a}$ & 一点庐 & & & \\
\hline 听不到] $=>$ [声 & 听不至 & & & \\
\hline [一点, 风] $=>$ [声音] [ & 一点应 & & & \\
\hline [一点, 听不到] $=>$ [声 & 一点听入 & & & 38 \\
\hline [制暖, 听不到] => [声音] [heating, cannot hear] => [sound] & 听不到制暖声音/no & & & 12.044 \\
\hline $\begin{array}{l}\text { [风, 几乎, 听不到] => [声音] } \\
\text { [sound] }\end{array}$ & $\begin{array}{c}\text { 几乎听不到风的声音/hardly hear the } \\
\text { wind }\end{array}$ & 0.00 & 0.7321 & 12.39 \\
\hline
\end{tabular}

TABLE 6: Representative CRs.

\begin{tabular}{lcc}
\hline No. & CRs & Support \\
\hline$C_{1}$ & Being simple and liberal & 0.0513 \\
$C_{2}$ & High-tech and intelligence & 0.0391 \\
$C_{3}$ & Being fashionable and novel & 0.0291 \\
$C_{4}$ & Low noise & 0.0487 \\
$C_{5}$ & Energy conservation & 0.0559 \\
$C_{6}$ & Fast cooling and heating & 0.0828 \\
$C_{7}$ & Strong mode and soft wind & 0.0270 \\
$C_{8}$ & Safety protection & 0.0457 \\
$C_{9}$ & Mobile APP control & 0.0446 \\
\hline
\end{tabular}

reasonable response rate of 91 percent. Based on Definition 2 , the maximum of membership (satisfaction) is 0.9 and minimum of membership (dissatisfaction) is 0 ; thus, the CRs membership function can be obtained (Figure 2) with every interviewee's membership to standard answers via functions (16)-(20) and CR value $S F_{i}$ (Table 7) via functions (21)-(23). Besides, according to Chen [60], the adjustment coefficient of attractive quality, one-dimensional quality, must-be quality, and indifferent quality set is $4,2,1$, and 0 in $\mathrm{KM}$ and FKM, respectively.
4.3. Step 3. Obtaining CRs Weights. According to Table 6, we constructed CRs hierarchy process (Figure 6). 10 experts with more than 5-year development experience and 5-year design experience were selected to act as interviewees; thus relation matrices were obtained via pairwise comparison, and calculated intuitionistic preference matrices are of acceptable consistency via functions (24)-(27). Then, nonconsistency relation matrices were calculated via (28)-(30). The consistency threshold value $\tau$ was selected as 0.1 (Table 8) and controlling parameter $\sigma$ was set to 0.8 . The 1 st, 6 th, and 
TABLE 7: The comparative results of the case study.

\begin{tabular}{|c|c|c|c|c|c|c|c|c|c|c|c|c|c|c|}
\hline \multirow{2}{*}{ CRs } & \multicolumn{5}{|c|}{ Classical KM } & \multicolumn{5}{|c|}{ FKM } & \multicolumn{2}{|c|}{ CFKM } & \multicolumn{2}{|c|}{ IFKM } \\
\hline & M & $\mathrm{O}$ & A & I & Cat. & M & $\mathrm{O}$ & A & I & Cat. & Eval. & Pri. & Eval. & Pri. \\
\hline$C_{1}$ & 0 & 49 & 80 & 90 & I $(0)$ & 2 & 57 & 116 & 102 & A (4) & 0.2173 & 1 & 0.1973 & 1 \\
\hline$C_{2}$ & 6 & 15 & 54 & 111 & I $(0)$ & 7 & 25 & 50 & 138 & I $(0)$ & 0.0912 & 3 & 0.1393 & 3 \\
\hline$C_{3}$ & 0 & 11 & 77 & 97 & I $(0)$ & 54 & 4 & 103 & 116 & I $(0)$ & 0.1425 & 2 & 0.1500 & 2 \\
\hline$C_{4}$ & 1 & 12 & 79 & 94 & I $(0)$ & 77 & 15 & 43 & 46 & M (1) & 0.2736 & 3 & 0.2696 & 1 \\
\hline$C_{5}$ & 2 & 12 & 64 & 101 & I $(0)$ & 80 & 52 & 51 & 31 & M (1) & 0.3312 & 1 & 0.2481 & 2 \\
\hline$C_{6}$ & 1 & 4 & 48 & 124 & I $(0)$ & 77 & 24 & 58 & 49 & M (1) & 0.3130 & 2 & 0.2088 & 3 \\
\hline$C_{7}$ & 0 & 2 & 42 & 144 & I $(0)$ & 57 & 49 & 36 & 92 & I $(0)$ & 0.1376 & 5 & 0.1371 & 5 \\
\hline$C_{8}$ & 13 & 2 & 37 & 138 & I $(0)$ & 41 & 55 & 29 & 71 & I $(0)$ & 0.2084 & 4 & 0.1652 & 4 \\
\hline$C_{9}$ & 0 & 11 & 62 & 113 & I (0) & 21 & 91 & 37 & 58 & $\mathrm{O}(2)$ & 0.1359 & 6 & 0.1296 & 6 \\
\hline
\end{tabular}

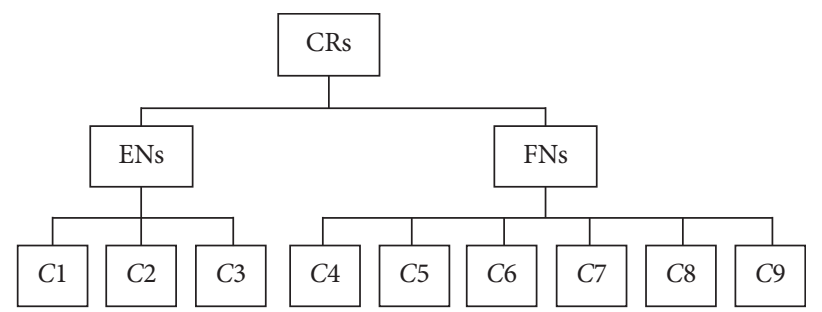

FIgURE 6: Hierarchical structure of the case study.

TABle 8: Consistency test of IFAHP.

\begin{tabular}{lcccc}
\hline Experts & First & & & Second \\
& ENs threshold & FNs threshold & ENs threshold & FNs threshold \\
\hline 1 & 0.0000 & 0.0767 & 0.0628 & - \\
2 & 0.3087 & 0.0915 & 0.0628 & - \\
3 & 0.3087 & 0.0563 & 0.0541 & - \\
4 & 0.2923 & 0.0422 & 0.0508 & - \\
5 & 0.2632 & 0.0716 & - & - \\
6 & 0.0000 & 0.0965 & 0.0159 & - \\
8 & 0.0429 & 0.0966 & 0.0240 & - \\
9 & 0.1032 & 0.0278 & 0.0235 & - \\
\hline
\end{tabular}

7th experts make acceptable consistency in ENs, and all experts make acceptable consistency in FNs. At last, we fused all the weights from the lowest level to the highest level via functions (31-32) (Table 9).

4.4. Step 4. Obtaining ECs Weights and Goals. Most of VACs consist of refrigeration cycle system, air circulation system, electrical control system, and shell system (Table 10). We calculated the adjusted weights obtained by formula (33) and collected relation matrixes of HOWs and WHATs from 10 experts and converted them into IFVs via Table 3 . Then, the target satisfaction was set through interview in 3000-4000 RMB VAC; the rate of satisfaction improvement is shown via (34); the absolute weights are gained by formula (35). Thus, we obtained the standard weights of $E C N_{j}$ via functions (36)(38) and HOQ of VAC (Figure 7). At last, according to technical benchmarks, goals were established in NPDD.

\section{Comparative Analysis}

Comparing the result weights of CRs in AHP, FAHP, and IFAHP (Table 11), the weights are similar; FNs are higher than ENs, and the results of FAHP are quite different between AHP and IFAHP. However, in the IFAHP, if we do not consider the nonmembership degree of $W K_{j}$ when ranking the priority, the result of the IFAHP method will be ENs $>$ FNs as well because $0.0469>0.0438>0.0338>0.0094$ $\ldots>0.0052$, which is consistent with the result of the FAHP method. The different results imply that the IFVs can represent the preferences of the pairwise comparison more comprehensively.

Comparing adjustment coefficient in KM, FKM, CFKM, and IFKM (Table 7), the classical Kano model does not work well in this case study, and FKM is worse than CFKM and IFKM in rationality of the classification results of CRs. The CFKM and IFKM have similar results, and the 
TABLE 9: Weights of IFAHP.

\begin{tabular}{lccrr}
\hline Criterion & $W_{i}$ & Subcriterion & $W_{i j}$ & $W K_{i}$ \\
\hline \multirow{2}{*}{ ENs } & & $\mathrm{C}_{1}$ & $(0.0957,0.5974)$ & $(0.0338,0.8589)$ \\
& $(0.3529,0.6494)$ & $\mathrm{C}_{2}$ & $(0.1241,0.5375)$ & $(0.0438,0.8379)$ \\
& & $\mathrm{C}_{3}$ & $(0.1328,0.7034)$ & $(0.0469,0.8960)$ \\
& & $\mathrm{C}_{4}$ & $(0.0183,0.7598)$ & $(0.0094,0.8710)$ \\
FNs & & $\mathrm{C}_{5}$ & $(0.0183,0.7598)$ & $(0.0094,0.8713)$ \\
& $(0.5158,0.4643)$ & $\mathrm{C}_{6}$ & $(0.0151,0.7998)$ & $(0.0078,0.8927)$ \\
& & $\mathrm{C}_{7}$ & $(0.0133,0.8159)$ & $(0.0069,0.9014)$ \\
& & $\mathrm{C}_{8}$ & $(0.0153,0.7895)$ & $(0.0079,0.8873)$ \\
& & $\mathrm{C}_{9}$ & $(0.0100,0.8489)$ & $(0.0052,0.9190)$ \\
\hline
\end{tabular}

TABLe 10: ECs of VAC.

\begin{tabular}{lccc}
\hline Refrigeration cycle system & Air circulation system & Electrical control system & Shell system \\
\hline Refrigeration cycle power parts $\left(A_{1}\right)$ & Forming airflow parts $\left(A_{4}\right)$ & Information display $\left(A_{7}\right)$ & Body $\left(A_{9}\right)$ \\
Thermal exchange parts $\left(A_{2}\right)$ & Air charge parts $\left(A_{5}\right)$ & Intelligent control parts $\left(A_{8}\right)$ & Top $\left(A_{10}\right)$ \\
$\begin{array}{l}\text { Refrigerant flow and pressure parts }\left(A_{3}\right) \\
\end{array}$ & Air flow direction controller parts $\left(A_{6}\right)$ & Base $\left(A_{11}\right)$ & Air outlet grille $\left(A_{12}\right)$ \\
\hline
\end{tabular}

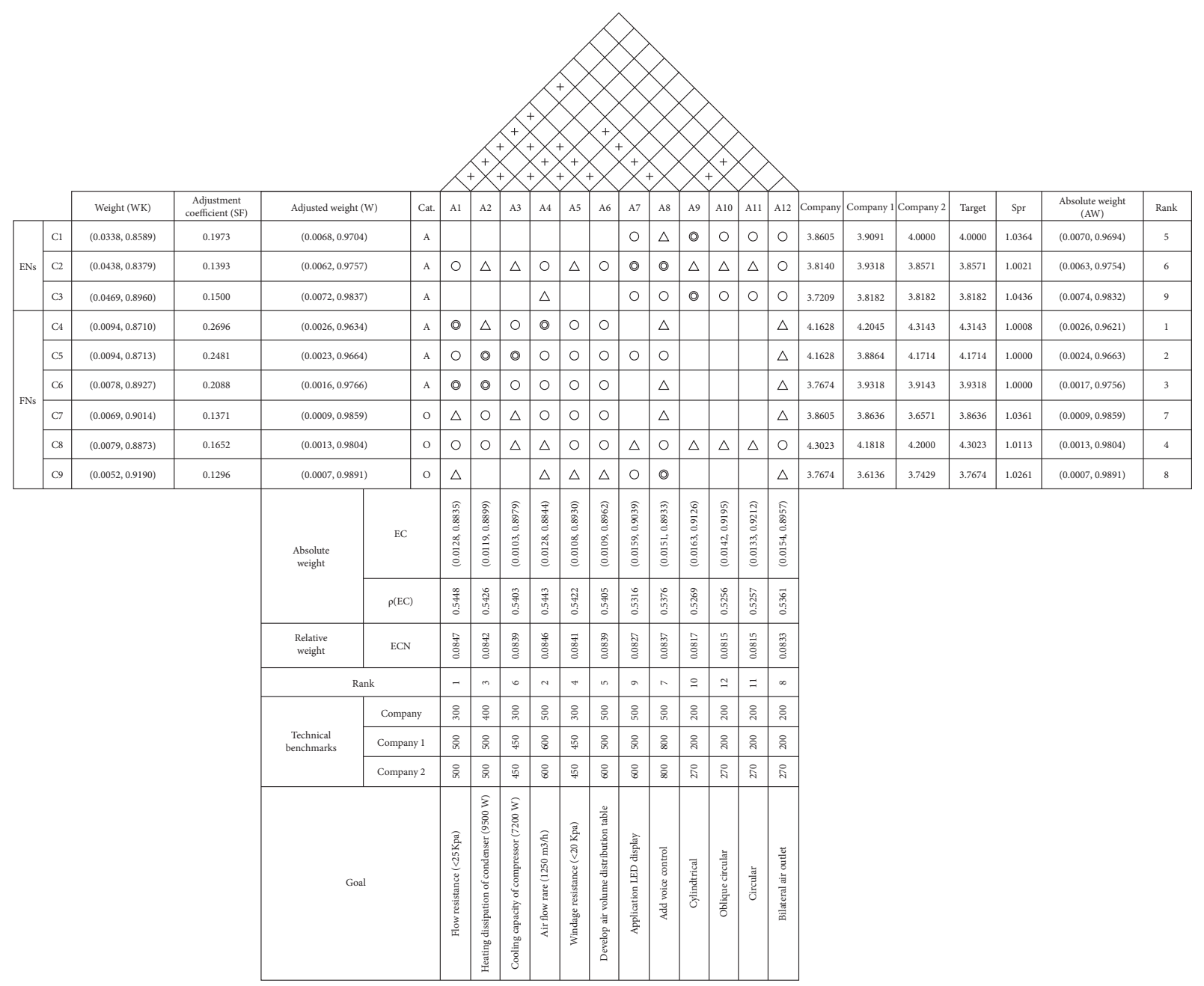

Figure 7: HOQ case study. 
TABLE 11: Weight comparison.

\begin{tabular}{|c|c|c|c|c|c|c|}
\hline & AHP & Pri. & FAHP & Pri. & IFAHP & Pri. \\
\hline$C_{1}$ & 0.0502 & 7 & 0.1563 & 2 & $(0.0338,0.8589)$ & 7 \\
\hline$C_{2}$ & 0.1250 & 4 & 0.1686 & 1 & $(0.0438,0.8379)$ & 8 \\
\hline$C_{3}$ & 0.0247 & 9 & 0.1363 & 3 & $(0.0469,0.8960)$ & 9 \\
\hline$C_{4}$ & 0.1863 & 2 & 0.0975 & 4 & $(0.0094,0.8710)$ & 1 \\
\hline$C_{5}$ & 0.2328 & 1 & 0.0969 & 5 & $(0.0094,0.8713)$ & 2 \\
\hline$C_{6}$ & 0.1127 & 5 & 0.0894 & 6 & $(0.0078,0.8927)$ & 4 \\
\hline$C_{7}$ & 0.0728 & 6 & 0.0861 & 8 & $(0.0069,0.9014)$ & 5 \\
\hline$C_{8}$ & 0.1498 & 3 & 0.0891 & 7 & $(0.0079,0.8873)$ & 3 \\
\hline$C_{9}$ & 0.0455 & 8 & 0.0798 & 9 & $(0.0052,0.9110)$ & 6 \\
\hline
\end{tabular}

TABLE 12: Absolute weight comparison.

\begin{tabular}{|c|c|c|c|c|c|}
\hline & Rsi & AHP ${ }^{*} \mathrm{KM}^{*} \mathrm{Rsi}$ & FAHP *FKM * Rsi & IFAHP * IFKM * Rsi & Pri. \\
\hline$\overline{C_{1}}$ & 1.0361 & 0.0000 & 0.6478 & $(0.0070,0.9694)$ & 5 \\
\hline $\mathrm{C}_{2}$ & 1.0113 & 0.0000 & 0.0000 & $(0.0063,0.9754)$ & 6 \\
\hline$C_{3}$ & 1.0261 & 0.0000 & 0.0000 & $(0.0074,0.9832)$ & 9 \\
\hline$C_{4}$ & 1.0364 & 0.0000 & 0.1010 & $(0.0026,0.9621)$ & 1 \\
\hline$C_{5}$ & 1.0021 & 0.0000 & 0.0971 & $(0.0024,0.9663)$ & 2 \\
\hline $\mathrm{C}_{6}$ & 1.0436 & 0.0000 & 0.0933 & $(0.0017,0.9756)$ & 3 \\
\hline$C_{7}$ & 1.0008 & 0.0000 & 0.0000 & $(0.0009,0.9859)$ & 7 \\
\hline$C_{8}$ & 1.0000 & 0.0000 & 0.0000 & $(0.0013,0.9804)$ & 4 \\
\hline$C_{9}$ & 1.0000 & 0.0000 & 0.1596 & $(0.0007,0.9891)$ & 8 \\
\hline
\end{tabular}

difference between the two methods is mainly in the attractive and one-dimensional qualities which are located in the cells $(1,4)$ and $(1,5)$, respectively, because the IFS is more comprehensive and flexible than FS in describing uncertainty.

According to Chen [60], the adjustment coefficient of attractive quality, one-dimensional quality, must-be quality, and indifferent quality set is $4,2,1$, and 0 in $\mathrm{KM}$ and FKM, respectively. The absolute weight of CRs is done by comparison in Table 12; there are limitations which ignore the difference of the satisfaction coefficient between the consumer demand items and the same quality attributes. Besides, the absolute weight cannot obtain good performance in HOQ which used KM and FKM as adjustment coefficient.

\section{Discussion}

In the classical Kano model, designers should follow the quality ranking for NPDD, which is mustbe $>$ attractive $>$ one-dimensional $>$ indifferent. One strategy is to integrate all the must-be, one-dimensional, and attractive quality into the product. However, the resource is quite limited; thus it is hard for them to decide which four of the six attractive qualities should be included with the addition of other qualities. In consequence, we proposed that IFKM can obtain CR preference, which includes affirmation, negation, hesitation, and priority, which effectively express VOC. In this paper, we chose VAC as an example. The ENs' priority of KM category (Table 7) is simple and liberal $>$ fashionable and novel $>$ high-tech and intelligence, and all of them belong to attractive quality. The FNs' priority of category is low noise > energy conservation $>$ fast cooling and heating, which are attractive quality; the order of one- dimensional quality is safety protection $>$ strong mode and soft wind > mobile APP control.

In order to obtain and compare the weight of the ENs and FNs, we conducted IFAHP process that analyzed overall weights about CRs and multiplies the adjustment coefficient via IFKM. Thus, we got the adjusted weights in the field of VAC's NPDD. The absolute weights should contain adjusted weights and ratios of satisfaction, which based on competition benchmarks (Figure 7). Thus, the result of absolute weights pirority is low noise $>$ energy saving $>$ fast cooling and heating $>$ safety protection $>$ simple and liberal $>$ hightech and intelligence $>$ strong mode and soft wind $>$ mobile APP control > fashion and novel. According to IFKM category and resource constraint, the strategy of this company should choose low noise, energy conservation, fast cooling and heating, and being simple and liberal as the attractive quality, finally with the addition of other qualities. Because the attractive quality massively improves CS (Figure 1) and the one-dimensional quality has linear relation to CS.

The refrigeration cycle power parts and forming airflow parts have the highest priority via IFQFD, because of low noise and energy conservation, which are the main sources of sound and power consumption, being the top two of CRs. And the thermal exchange part is the third importance ECs, because it has strong relation to energy conservation and fast cooling and heating. In additional, the ranking of the ECs of refrigeration cycle system, air circulation system, and electrical control system is higher than that of shell system; thus it is consistent with the FNs being larger than the ENs. Thus, this research has high credibility in CS analysis and can provide effective support for decision-making. Finally, the company established development goals based on technical benchmarks. 


\section{Conclusions}

In this study, we proposed a new method for NPDD based on text mining, CS, and IFS. We applied text mining technique to retrieve CRs from big data and combined this with advantage of IFS which can better process uncertain and hesitant information, to integrate Kano model, AHP, and QFD methods. Thus, we can establish goals about the customer-oriented NPDD. In additional, text mining can directly obtain expected information from unstructured data and provide a scientific basis for NPDD. The IFSs have more flexible and comprehensive features in describing objective phenomena than classical FS. And the evaluation of various preference types is unified into IFS based on the heterogeneous characteristics of participants that can minimize the loss of decision information.

Since certain limitations still exist, this research needs to be continually improved. First, it is necessary to develop a supervised algorithm for the extraction of CRs with low frequency in text mining, because lower frequency of CRs like dehumidification and air purification will be affected by geographical and environment factors. Second, the impact of multiple Kansei images of product on customers satisfaction remains to be studied, which means that a product may contain factors like being fashionable, elegant, simple, beautiful, etc. and generally have nonlinear relationship to product form. Third, the threshold $(\phi)$ of the $S F$ is determined by practitioners based on experience. There is a need to develop an approach to determine the suitable values for decision-making.

\section{Data Availability}

The data used to support the findings of this study are available from the corresponding author upon request.

\section{Conflicts of Interest}

The authors declare that there are no conflicts of interest regarding the publication of this paper.

\section{Acknowledgments}

This research was supported by the Shanghai Summit Discipline in Design of China and Shanghai Philosophy and Social Science Planning Project (Grant nos. DC17014 and 2019ZJX002).

\section{References}

[1] E. W. Anderson and V. Mittal, "Strengthening the satisfaction-profit chain," Journal of Service Research, vol. 3, no. 2, pp. 107-120, 2000.

[2] M. Hu and B. Liu, "Mining and summarizing customer reviews," in Proceedings of the 10th ACM SIGKDD International Conference on Knowledge Discovery and Data Mining, pp. 168-177, Seattle, WA, USA, August 2004.

[3] A. J. Newman and D. Patel, "The marketing directions of two fashion retailers," European Journal of Marketing, vol. 38, no. 7, pp. 770-789, 2004.
[4] M. Nagamachi, "Kansei engineering as a powerful consumeroriented technology for product development," Applied Ergonomics, vol. 33, no. 3, pp. 289-294, 2002.

[5] M. Nagamachi, "Kansei Engineering: a new ergonomic consumer-oriented technology for product development," International Journal of Industrial Ergonomics, vol. 15, no. 1, pp. 3-11, 1995.

[6] S.-M. Yang, M. Nagamachi, and S.-Y. Lee, "Rule-based inference model for the Kansei engineering system," International Journal of Industrial Ergonomics, vol. 24, no. 5, pp. 459-471, 1999.

[7] H. M. Khalid and M. G. Helander, "Customer emotional needs in product design," Concurrent Engineering, vol. 14, no. 3, pp. 197-206, 2006.

[8] S. Yang, M. Nagamachi, and S. Lee, "Rule-based inference model for the Kansei engineering system," International Journal of Industrial Ergonomics, vol. 24, no. 5, pp. 459-471, 2011.

[9] C.-C. Yang, "Constructing a hybrid Kansei engineering system based on multiple affective responses: application to product form design," Computers and Industrial Engineering, vol. 60, no. 4, pp. 760-768, 2011.

[10] M.-D. Shieh and Y.-E. Yeh, "Developing a design support system for the exterior form of running shoes using partial least squares and neural networks," Computers and Industrial Engineering, vol. 65, no. 4, pp. 704-718, 2013.

[11] C.-H. Wang, "Integrating Kansei engineering with conjoint analysis to fulfil market segmentation and product customisation for digital cameras," International Journal of Production Research, vol. 53, no. 8, pp. 2427-2438, 2015.

[12] C. H. Papadimitriou, P. Raghavan, H. Tamaki et al., "Latent semantic indexing: a probabilistic analysis," Journal of Computer and System Sciences, vol. 61, no. 2, pp. 217-235, 1998.

[13] T. Hofmann, "Probabilistic latent semantic indexing," in Proceedings of the 22nd Annual International ACM SIGIR Conference on Research and Development in Information Retrieval, pp. 50-57, Berkeley, CA, USA, August 1999.

[14] D. M. Blei, A. Y. Ng, and M. I. Jordan, "Latent dirichlet allocation," The Journal of Machine Learning Research, vol. 3, pp. 993-1022, 2003.

[15] D. Walter, Y. Ophir, and K. H. Jamieson, "Russian twitter accounts and the partisan polarization of vaccine discourse, 2015-2017," American Journal of Public Health, vol. 110, no. 5, pp. 718-724, 2020.

[16] D. Maier, A. Waldherr, P. Miltner et al., "Applying LDA topic modeling in communication research: toward a valid and reliable methodology," Communication Methods and Measures, vol. 12, no. 2-3, pp. 93-118, 2018.

[17] X. Wei X and W. Croft, "LDA-based document models for Ad-hoc retrieval," in Proceedings of the 29th Annual International ACM SIGIR Conference on Research and Development in Information Retrieval, pp. 178-185, Seattle, WA, USA, August 2006.

[18] R. Agrawal and R. Srikant, "Fast algorithms for mining association rules," in Proceedings of the 20th International Conference on Very Large Data Bases, vol. 1215, pp. 487-499, Santiago, SM, Chile, September 1994.

[19] Y. Guo, M. Wang, and X. Li, "Application of an improved Apriori algorithm in a mobile e-commerce recommendation system," Industrial Management \& Data Systems, vol. 117, no. 2, pp. 287-303, 2017.

[20] S. Panjaitan, M. Amin, S. Lindawati et al., "Implementation of apriori algorithm for analysis of consumer purchase patterns," 
Journal of Physics: Conference Series, IOP Publishing, vol. 1255, no. 1, Article ID 012057, 2019.

[21] Y. Liu, "Study on application of apriori algorithm in data mining," in Proceedings of the 2010 Second International Conference on Computer Modeling and Simulation, vol. 3, pp. 111-114, Sanya, HN, China, January 2010.

[22] N. Kano, N. Seraku, and F. Takahashi, "Attractive quality and must-be quality," Journal of the Japanese Society for Quality Control, vol. 14, no. 2, pp. 39-48, 1984.

[23] K. Matzler and H. H. Hinterhuber, "How to make product development projects more successful by integrating Kano's model of customer satisfaction into quality function deployment," Technovation, vol. 18, no. 1, pp. 25-38, 1998.

[24] K. C. Tan and X. X. Shen, "Integrating Kano's model in the planning matrix of quality function deployment," Total Quality Management, vol. 11, no. 8, pp. 1141-1151, 2000.

[25] Q. Xu, R. J. Jiao, X. Yang, M. Helander, H. M. Khalid, and A. Opperud, "An analytical Kano model for customer need analysis,” Design Studies, vol. 30, no. 1, pp. 87-110, 2009.

[26] P. Földesi, L. T. Kóczy, and J. Botzheim, "Fuzzy extension for Kano's model using bacterial evolutionary algorithm," in Proceedings of the 2007 International Symposium on Computational Intelligence and Intelligent Informatics, pp. 147151, Agadir, SM, Morocco, March 2007.

[27] Y.-C. Lee and S.-Y. Huang, "A new fuzzy concept approach for Kano's model," Expert Systems with Applications, vol. 36, no. 3, pp. 4479-4484, 2009.

[28] M. Wu and L. Wang, "A continuous fuzzy Kano's model for customer requirements analysis in product development," Proceedings of the Institution of Mechanical Engineers, Part B: Journal of Engineering Manufacture, vol. 226, no. 3, pp. 535-546, 2012.

[29] T. L. Satty, The Analysis Hierarchy Process, McGraw-Hill, New York, NY, USA, 1980.

[30] P. J. M. van. Laarhoven and W. Pedrycz, "A fuzzy extension of Saaty's priority theory," Fuzzy Sets and Systems, vol. 11, no. 1, pp. 229-241, 1983.

[31] M. Kwiesielewicz, "A note on the fuzzy extension of Saaty's priority theory," Fuzzy Sets and Systems, vol. 95, no. 2, pp. 161-172, 1998.

[32] C. G. E. Boender, J. G. de Graan, and F. A. Lootsma, "Multicriteria decision analysis with fuzzy pairwise comparisons," Fuzzy Sets and Systems, vol. 29, no. 2, pp. 133-143, 1989.

[33] Z. Xu and H. Liao, "Intuitionistic fuzzy analytic hierarchy process," IEEE Transactions on Fuzzy Systems, vol. 22, no. 4, pp. 749-761, 2014.

[34] S.-W. Hsiao and Y.-C. Ko, "A study on bicycle appearance preference by using FCE and FAHP," International Journal of Industrial Ergonomics, vol. 43, no. 4, pp. 264-273, 2013.

[35] K. Kamvysi, K. Gotzamani, A. Andronikidis, and A. C. Georgiou, 'Capturing and prioritizing students' requirements for course design by embedding Fuzzy-AHP and linear programming in QFD," European Journal of Operational Research, vol. 237, no. 3, pp. 1083-1094, 2014.

[36] K. Chakraborty, S. Mondal, and K. Mukherjee, "Analysis of product design characteristics for remanufacturing using Fuzzy AHP and axiomatic design," Journal of Engineering Design, vol. 28, no. 5, pp. 338-368, 2017.

[37] Y. Akao, "Development history of quality function deployment," The Customer Driven Approach to Quality Planning and Deployment, vol. 339, p. 90, 1994.

[38] J. R. Hauser, "The house of quality," Harvard Business Review, vol. 66, no. 3, pp. 63-73, 1988.
[39] X. Lai, M. Xie, and T. C. Tan, "Optimizing product design using the Kano model and QFD," in Proceedings of the 2004 IEEE International Engineering Management Conference, vol. 3, pp. 1085-1089, Singapore, October 2004.

[40] N. Tu, T. Zhang, Q.-Y. He, H.-F. Zhang, and Y.-H. Li, "Applying combined AHP-QFD method in new product development: a case study in developing new sports earphone," in Proceedings of the 2011 International Conference on Management Science \& Industrial Engineering, Harbin, HL, China, January 2011.

[41] G. Bayraktaroğlu and O. Özge, "Integrating the Kano model, AHP and Planning Matrix: QFD application in library services," Library Management, vol. 29, no. 45, pp. 327-351, 2008.

[42] L. P. Khoo and N. C. Ho, "Framework of a fuzzy quality function deployment system," International Journal of Production Research, vol. 34, no. 2, pp. 299-311, 1996.

[43] Q. Wu, "Fuzzy measurable house of quality and quality function deployment for fuzzy regression estimation problem," Expert Systems with Applications, vol. 38, no. 12, pp. 14398-14406, 2011.

[44] I. Mahdavi, N. Mahdavi-Amiri, A. Heidarzade, and R. Nourifar, "Designing a model of fuzzy TOPSIS in multiple criteria decision making," Applied Mathematics and Computation, vol. 206, no. 2, pp. 607-617, 2008.

[45] T. Aouam, S. I. Chang, and E. S. Lee, "Fuzzy MADM: an outranking method," European Journal of Operational Research, vol. 145, no. 2, pp. 317-328, 2003.

[46] S. J. Chen and C. L. Hwang, Fuzzy Multiple Attribute Decision Making, Springer, Berlin, Heidelberg, Germany, 1992.

[47] K. T. Atanassov, "Intuitionistic fuzzy sets," Fuzzy Sets and Systems, vol. 20, no. 1, pp. 87-96, 1986.

[48] K. T. Atanassov, Intuitionistic Fuzzy Sets: Theory and Applications, Springer, Berlin, Heidelberg, Germany, 1999.

[49] K. T. Atanassov, On Intuitionistic Fuzzy Sets Theory, Springer, Germany, 2012.

[50] D. F. Li, J. X. Nan, and M. J. Zhang, "A ranking method of triangular intuitionistic fuzzy numbers and application to decision making," International Journal of Computational Intelligence Systems, vol. 3, no. 5, pp. 522-530, 2010.

[51] D.-F. Li, "A ratio ranking method of triangular intuitionistic fuzzy numbers and its application to MADM problems," Computers and Mathematics with Applications, vol. 60, no. 6, pp. 1557-1570, 2010.

[52] D.-F. Li, "Multiattribute decision making models and methods using intuitionistic fuzzy sets," Journal of Computer and System Sciences, vol. 70, no. 1, pp. 73-85, 2005.

[53] E. Szmidt and J. Kacprzyk, "Amount of information and its reliability in the ranking of atanassov's intuitionistic fuzzy alternatives," Studies in Computational Intelligence, vol. 222, pp. 7-19, 2009.

[54] Z. S. Xu, "Intuitionistic fuzzy aggregation operators," Fuzzy System, vol. 15, no. 6, pp. 1179-1187, 2007.

[55] Z. Xu, "Intuitionistic preference relations and their application in group decision makingts," Information Sciences, vol. 177, no. 11, pp. 2363-2379, 2007.

[56] M. Rosen-Zvi, T. L. Griffiths, M. Steyvers et al., "The authortopic model for authors and documents," in Proceedings of the 20th Conference in Uncertainty in Artificial Intelligence, Banff, AB, Canada, July, 2004.

[57] C. Berger, R. E. Blauth, and D. Boger, "Kano's methods for understanding customer-defined quality," Computer Science, vol. 2, no. 4, pp. 3-35, 1995. 
[58] L.-H. Chen and W.-C. Ko, "A fuzzy nonlinear model for quality function deployment considering Kano's concept," Mathematical and Computer Modelling, vol. 48, no. 3-4, pp. 581-593, 2008.

[59] L. Cohen, Quality Function Deployment: How to Make QFD Work for You, Addison-Wesley, Boston, MA, USA, 1995.

[60] C.-C. Chen and M.-C. Chuang, "Integrating the Kano model into a robust design approach to enhance customer satisfaction with product design," International Journal of Production Economics, vol. 114, no. 2, pp. 667-681, 2008. 